\title{
Non-contact thrust stand calibration method for repetitively-pulsed electric thrusters
}

\author{
Andrea R. Wong, Alexandra Toftul $†$ Kurt A. Polzin $\ddagger$ and J. Boise Pearson ${ }^{\S}$ \\ NASA-Marshall Space Flight Center, Huntsville, AL 35812
}

\begin{abstract}
A thrust stand calibration technique for use in testing repetitively-pulsed electric thrusters for in-space propulsion has been developed and tested using a modified hanging pendulum thrust stand. In the implementation of this technique, current pulses are applied to a solenoidal coil to produce a pulsed magnetic field that acts against the magnetic field produced by a permanent magnet mounted to the thrust stand pendulum arm. The force on the magnet is applied in this non-contact manner, with the entire pulsed force transferred to the pendulum arm through a piezoelectric force transducer to provide a time-accurate force measurement. Modeling of the pendulum arm dynamics reveals that after an initial transient in thrust stand motion the quasisteady average deflection of the thrust stand arm away from the unforced or 'zero' position can be related to the average applied force through a simple linear Hooke's law relationship. Modeling demonstrates that this technique is universally applicable except when the pulsing period is increased to the point where it approaches the period of natural thrust stand motion. Calibration data were obtained using a modified hanging pendulum thrust stand previously used for steady-state thrust measurements. Data were obtained for varying impulse bit at constant pulse frequency and for varying pulse frequency. The two data sets exhibit excellent quantitative agreement with each other as the constant relating average deflection and average thrust match within the errors on the linear regression curve fit of the data. Quantitatively, the error on the calibration coefficient is roughly $1 \%$ of the coefficient value.
\end{abstract}

\section{Introduction}

$\mathrm{T}$ HE evaluation of the performance of any propulsion system requires the accurate measurement of thrust. While chemical rocket thrust is typically measured using a load cell, ${ }^{1}$ the low thrust levels associated with electric propulsion (EP) systems for in-space propulsion necessitate the use of much more sensitive measurement techniques. The problem is compounded when a pulsed thruster is tested, as it subjects the thrust measurement device to an unsteady force. Work by Cubbin et al. $,^{2} \mathrm{Haag},{ }^{3}$ and Markusic et al. ${ }^{4}$ present pulsed thrust measurement results obtained using torsional thrust stands where the pulsed thruster applied an impulse bit (impulse per pulse) to a swinging-gate thrust stand arm. In all three works the thrust balance response was calibrated by imparting an impulsive force (on the time scale of thrust stand motion) to the movable thrust stand arm using a swinging mass. The impulsive force imparted was found in Ref. [3] by calculating the momentum transfer during the impact from the measured change in velocity of the swinging mass and in Refs. [2,4] by directly measuring the imparted force using a piezoelectric force transducer. Knowing the thrust balance response to measured impulsive forces permitted the determination of a pulsed thruster's unknown impulse bit by measuring the response of the thrust balance. Typically pulsed thrust measurements have been performed in single-pulse mode (single-shot) only, though Haag also performed repetition-rate thrust measurements by pulsing a LES-8/9 pulsed plasma thruster (PPT) on a timescale much shorter than the response time of the thrust balance arm, measuring the average displacement of the arm during pulsing, and equating that displacement to a 'steady-state' average thrust level. ${ }^{3}$

In this paper, we describe a non-contact pulsed thrust calibration technique that is capable of electromagnetically applying a series of impulsive pulses to a thrust balance. The technique is capable of applying a single-impulse force for single-shot thrust measurement calibrations or providing multiple pulses to calibrate for a thruster operating

\footnotetext{
*Undergraduate Intern; currently undergraduate student, University of California-San Diego, La Jolla, CA.

$\dagger$ Undergraduate Intern; currently undergraduate student, University of Nebraska-Lincoln, Lincoln, NE.

$\ddagger$ Propulsion Research Engineer, Propulsion Research and Technology Applications Branch, Propulsion Systems Department. Senior Member AIAA.

$\S$ Nuclear Power Systems Team Lead, Propulsion Research and Technology Applications Branch, Propulsion Systems Department.
} 
in a repetition-rate mode. While the technique of performing data acquisition and analysis on a single-shot test is well developed, the problem of operating and performing measurements on a thruster in repetition-rate mode has not been examined in great detail, with only a few researchers measuring the average thrust stand deflection for repetitively-pulsed thrust measurements. The technique is tested using the Variable Amplitude Hanging Pendulum with Extended Range (VAHPER) thrust stand, ${ }^{5}$ demonstrating pulsed thrust measurement capabilities on this modified hanging-pendulum thrust stand that has only been used previously to measure the thrust levels of steady-state electric thrusters. ${ }^{6,7}$ The non-contact method is more easily controllable than the swinging impactor method used in previous works, applying impulsive forces to the stand in the same manner as the thruster. We also demonstrate in this paper that the measurement of the average thrust stand deflection during repetitively-pulsed calibration or operation is almost universally appropriate for the measurement of the average thrust and impulse bit, which for repetition-rate pulsing will obviate the need in the future to measure and anaylze the time-resolved motion of the thrust stand as is presently used when performing single pulse thrust stand measurements. ${ }^{2,4}$

The outline for the rest of this paper is as follows. In Section II we present an analytical model for thrust stand response to an arbitrary number of pulses. Section III contains a description of the test hardware used in the present effort. Data demonstrating the sensitivity and impulsive thrust measurement capabilities of the VAHPER thrust stand are presented in Sect. IV.

\section{Analytical Thrust Stand Response Model}

The response of most thrust stands, whether they are of the hanging or inverted pendulum or the swinging-gate variety, can be modeled as a torsional spring-mass-damper system. If the angular deflections are small, the deflection of the thrust stand arm can be linearized. For a repetitive, impulsive forcing function the governing equation can be written as

$$
\ddot{x}(t)+2 \omega_{n} \zeta \dot{x}(t)+\omega_{n}^{2} x(t)=\sum_{i=0}^{k} \frac{I_{\mathrm{bit}}}{m_{\mathrm{eff}}} \delta\left(t-\frac{i}{f}\right)
$$

where $x(t)$ represents thrust stand displacement as a function of time, $\omega_{n}$ is the natural frequency of the thrust stand, and $\zeta$ is the damping ratio. On the right side of the equation a repetitive impulsive forcing function with the magnitude $I_{\text {bit }} / m_{\text {eff }}$ is applied to the system to represent the pulsing thruster or calibration unit, where $I_{\text {bit }}$ is the magnitude of the impulse and $m_{\text {eff }}$ is the effective mass of the spring-mass-damper thrust stand system. The impulsive load is applied as a delta function, $\delta$ with a pulse width much less than the thrust stand natural period. This load is applied for a total of $k$ pulses at a pulse frequency $f$.

Solutions to the governing equation can be found for three separate cases, $\zeta<1, \zeta=1$, and $\zeta>1$, corresponding to the thrust stand being underdamped, critically damped, and overdamped, respectively. A short derivation of the solutions for each case for initial conditions $\dot{x}(t)=x(t)=0$ obtained using Laplace transforms can be found in the Appendix, while the more general solutions for an arbitrary set of initial conditions is given below as

$$
\begin{aligned}
& x(t)=\sum_{i=0}^{k}\left[\frac { I _ { \mathrm { bit } } } { 2 m _ { \mathrm { eff } } \omega _ { n } \sqrt { \zeta ^ { 2 } - 1 } } \operatorname { e x p } \{ - \omega _ { n } \zeta ( t - \frac { i } { f } ) \} \left(\exp \left\{\omega_{n} \sqrt{\zeta^{2}-1}\left(t-\frac{i}{f}\right)\right\}\right.\right. \\
& \left.\left.-\exp \left\{-\omega_{n} \sqrt{\zeta^{2}-1}\left(t-\frac{i}{f}\right)\right\}\right)\right] H\left(t-\frac{i}{f}\right) \\
& +\frac{x(0)}{2}\left[\left(\frac{-\zeta}{\sqrt{\zeta^{2}-1}}+1\right) \exp \left(-\omega_{n} \zeta+\omega_{n} t \sqrt{\zeta^{2}-1}\right)+\left(\frac{\zeta}{\sqrt{\zeta^{2}-1}}+1\right) \exp \left(-\omega_{n} \zeta-\omega_{n} t \sqrt{\zeta^{2}-1}\right)\right] \\
& +\left\{\frac{\dot{x}(0)+2 x(0) \omega_{n} \zeta}{2 \omega_{n} \sqrt{\zeta^{2}-1}}\right\} \exp \left(-\omega_{n} t \zeta\right)\left[\exp \left(\omega_{n} t \sqrt{\zeta^{2}-1}\right)-\exp \left(-\omega_{n} t \sqrt{\zeta^{2}-1}\right)\right] \\
& \text { for } \zeta>1 \\
& x(t)=\sum_{i=0}^{k}\left[\frac{I_{\mathrm{bit}}}{m_{\mathrm{eff}}}\left(t-\frac{i}{f}\right) \exp \left\{-\omega_{n}\left(t-\frac{i}{f}\right)\right\}\right] H\left(t-\frac{i}{f}\right)+x(0) \exp \left(-\omega_{n} t\right)\left[1+\omega_{n} t\right]+\dot{x}(0) t \exp \left(-\omega_{n} t\right) \\
& \text { for } \zeta=1
\end{aligned}
$$




$$
\begin{aligned}
& x(t)=\sum_{i=0}^{k}\left[\frac{I_{\mathrm{bit}}}{m_{\mathrm{eff}} \omega_{n} \sqrt{1-\zeta^{2}}} \exp \left\{-\omega_{n} \zeta\left(t-\frac{i}{f}\right)\right\} \sin \left\{\omega_{n} \sqrt{1-\zeta^{2}}\left(t-\frac{i}{f}\right)\right\}\right] H\left(t-\frac{i}{f}\right) \\
& +x(0) \exp \left(-\omega_{n} t \zeta\right)\left[\frac{-\zeta \sin \left(\omega_{n} t \sqrt{1-\zeta^{2}}\right)}{\sqrt{1-\zeta^{2}}}+\cos \left(\omega_{n} t \sqrt{1-\zeta^{2}}\right)\right] \\
& +\left[\frac{\dot{x}(0)+2 x(0) \omega_{n} \zeta}{\omega_{n} \sqrt{1-\zeta^{2}}}\right] \exp \left(-\omega_{n} t \zeta\right) \sin \left(\omega_{n} t \sqrt{1-\zeta^{2}}\right) \\
& \text { for } \zeta<1
\end{aligned}
$$

where $H$ is the Heaviside function.

As an illustrative example of the response, the solutions given in Eqs. (2), (3), and (4) with initial conditions $x(0)=0.5 \mathrm{~mm}$ and $\dot{x}(0)=2.5 \mathrm{~mm} / \mathrm{s}$ for a single pulse $(k=1)$ are given in Fig. 1a, showing the typical damped sinusoidal behavior of the system associated with an underdamped, critically damped, and overdamped system. Unless otherwise noted, all modeled solutions are for $I_{\mathrm{bit}}=10 \mathrm{mN}-\mathrm{s}, m_{\mathrm{eff}}=4.1 \mathrm{~kg}$, and $\omega_{n}=1.57 \mathrm{rad} / \mathrm{s}$. The time response of the system for an arbitrary number of pulses is presented in Fig. 1b for all three damping cases, again with illustrative initial conditions $x(0)=0.5 \mathrm{~mm}$ and $\dot{x}(0)=2.5 \mathrm{~mm} / \mathrm{s}$. We observe that after a brief transient period, the system begins to oscillates about a given average displacement value. The transient response for the stand is different for each damping case, but the long-term average displacement value about which the system oscillates is essentially unchanged in all three cases with nearly equivalent time-histories after the first few seconds. We also calculated the behavior for varying initial conditions and found that the long-term behavior of the system does not change for any initial conditions.
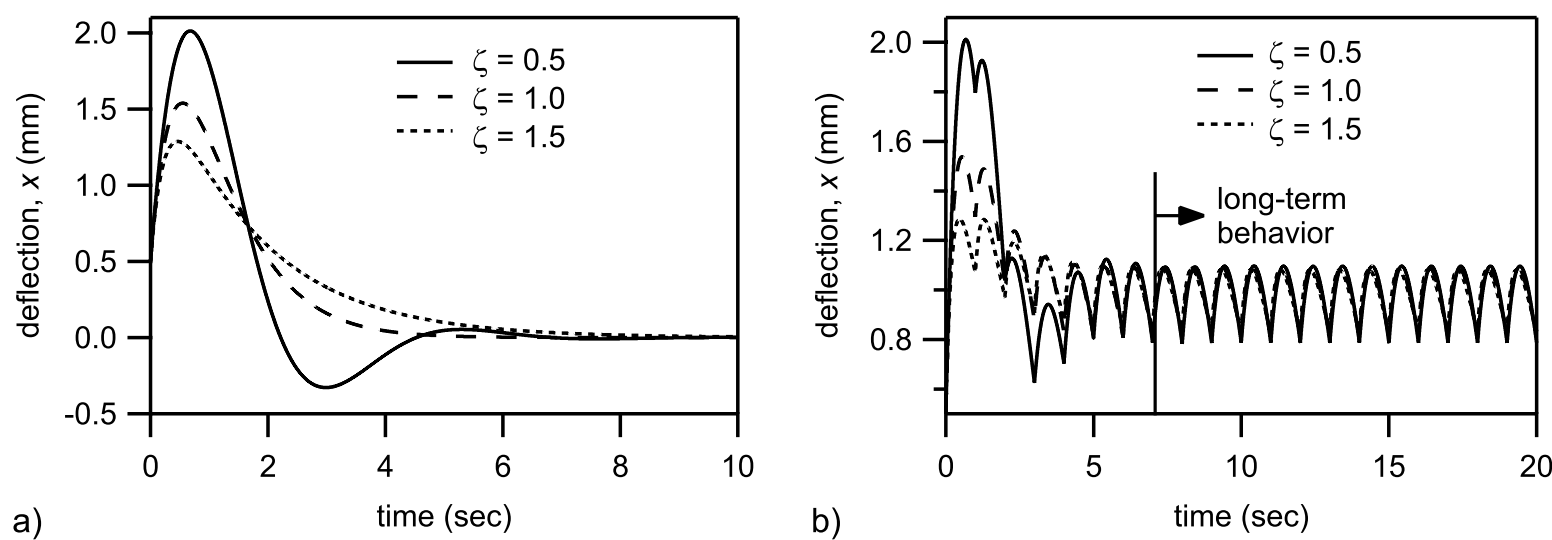

Figure 1. Response of the system described by Eq. (1) subject to the initial conditions $x(0)=0.5 \mathrm{~mm}$ and $\dot{x}(0)=\mathbf{2 . 5} \mathbf{~ m m} / \mathrm{s}$ for a) a single pulse and b) multiple pulses at a pulse frequency of $1 \mathrm{~Hz}$.

In the simulated response data we generated to demonstrate the efficacy of the pulsed calibration concept, the average applied force to the system described by Eq. (1) is equal to the impulse bit times the pulse frequency $\left(I_{\mathrm{bit}} \cdot f\right)$. If the relationship between this average applied force and the long-term average displacement value is linear, then a graph of the two should reveal the linear coefficient $k_{\text {eff }}$, which during testing would represent the calibration constant relating a measured average thrust stand deflection to the unknown impulse bit produced by a thruster. This spring constant $k_{\text {eff }}$ is related to the natural frequency and effective mass of the system as

$$
\omega_{n}^{2}=\frac{k_{\mathrm{eff}}}{m_{\mathrm{eff}}} .
$$

The deflection value is the difference between the zero position and the average value of the deflection once the response asymptotes to the long-term behavior observed in Fig. 1b. The average force can be adjusted by either varying $I_{\mathrm{bit}}$ or changing $f$. In Fig. 2a, $I_{\mathrm{bit}}$ is varied at a constant pulse frequency of $1 \mathrm{~Hz}$ to apply a range of forces to the system. The graph exhibits a linear relationship between the applied forces and the average displacements 
for all damping cases (only one case is shown for clarity since all three lie on top of each other). In Fig. 2b, the average force is changed by varying the pulse frequency at a constant impulse bit of $10 \mathrm{mN}$-s. We observe that the relationship between the applied force and the average displacement again follows a linear relationship, but only above the level where the pulse frequency at least twice as great as the system's natural frequency of $0.25 \mathrm{~Hz}$, with the discrepancy being most pronounced in the underdamped case. Physically, as the pulse frequency is lowered to this level the oscillator completes more than half a cycle between pulses and crosses zero, yielding a deflection response that is chaotic, never reaching the long-term repetitive pattern observed in Fig. 1b. The difference between the average value of the long-term deflection and zero was selected as the metric, as opposed to using the difference between the maximum or minimum long-term deflection value and zero, because the slope of the curves given in Fig. 2 using the average deflection metric best matches the value of $k_{\text {eff }}$ used in Eqs. (2), (3), and (4) to generate the deflection curves.
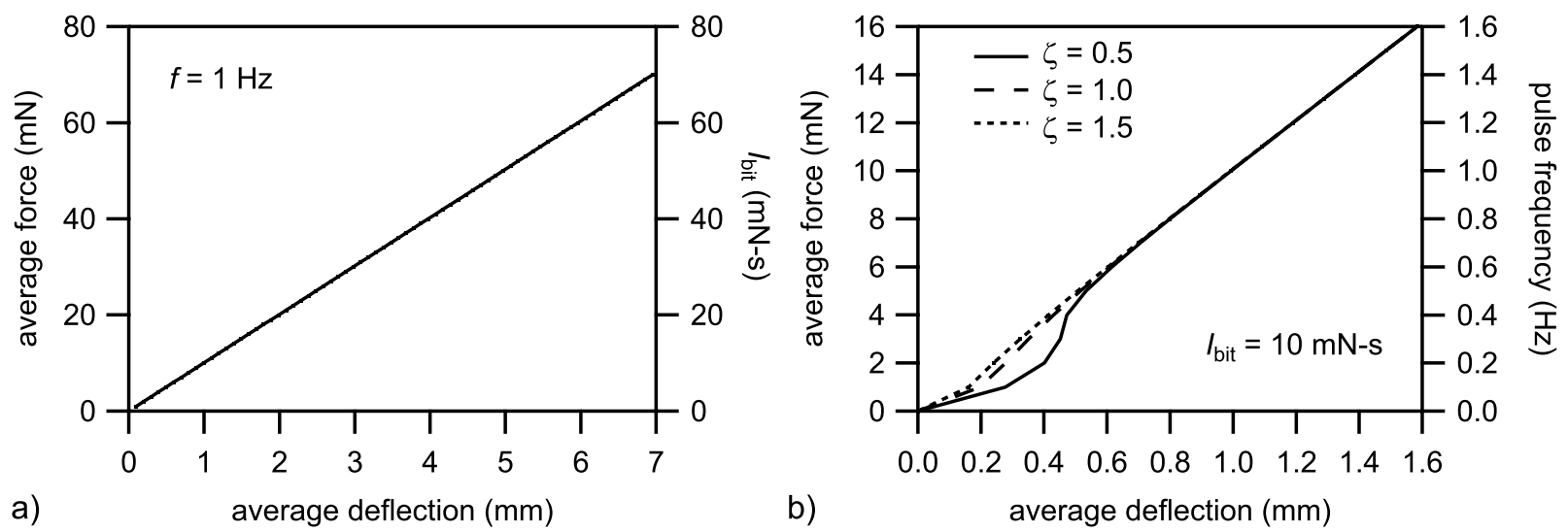

Figure 2. Average force applied to the system as a function of the average long-term deflection for a) varying impulse bit at a pulse frequency of $1 \mathrm{~Hz}$ and $\mathrm{b}$ ) varying pulse frequency at an impulse bit of $10 \mathrm{mN}-\mathrm{s}$.

\section{Experimental Apparatus}

A repetition-rate pulsed calibration was performed using the VAHPER thrust stand shown in Fig. 3a and previously described in Ref. [5]. The thrust stand is a variation on the hanging pendulum design, employing an adjustable mechanical linkage system to convert horizontal deflection of the pendulum arm to amplified vertical deflection of a secondary beam. The vertical deflection is measured using an optical linear gap displacement transducer (LGDT), while a passive eddy-current damping system is used to attenuate oscillatory motion of the balance mechanism. For the present work, a simulated pulsed inductive thruster having an approximate mass between 20-30 kg was mounted on the end of the pendulum arm to provide a realistic response of the thrust stand.

An impulsive force was imparted to the thrust stand pendulum arm using the apparatus shown photographically in Fig. 3b. In this apparatus, a power supply is connected in series with the solenoid and switched using a high-power MOSFET to repetitively apply current through the solenoid coil. A square-wave input pulse to the MOSFET yields an approximately square-wave current in the coil, which is fixed in position and produces a magnetic field that acts to repel the magnet. The magnet transmits an impulsive (on the time scale of thrust stand motion) force to the pendulum arm through a piezoelectric force transducer that records the applied time-varying force and possesses a resolution of $501.6 \mathrm{mV} / \mathrm{N}$. The force applied to the magnet, and consequently to the thrust stand pendulum arm, is transmitted in a non-contact manner through the magnetic field produced by the current flowing in the solenoid coil. The recorded force (integrated with respect to time to yield the impulse bit) and the measured deflection of the balance mechanism permit the experimental generation of calibration curves similar to those presented in Fig. 2.

\section{Calibration Data}

Thrust stand calibration data for repetitive pulsing are presented in this section demonstrating the applicability and usefulness of the analysis method outlined in Sect. II. Raw low-speed data showing the time-history of thrust stand deflection (given in $\mathrm{V}$ and acquired in the linear range of the position sensor) during a repetition-rate pulsed calibration 


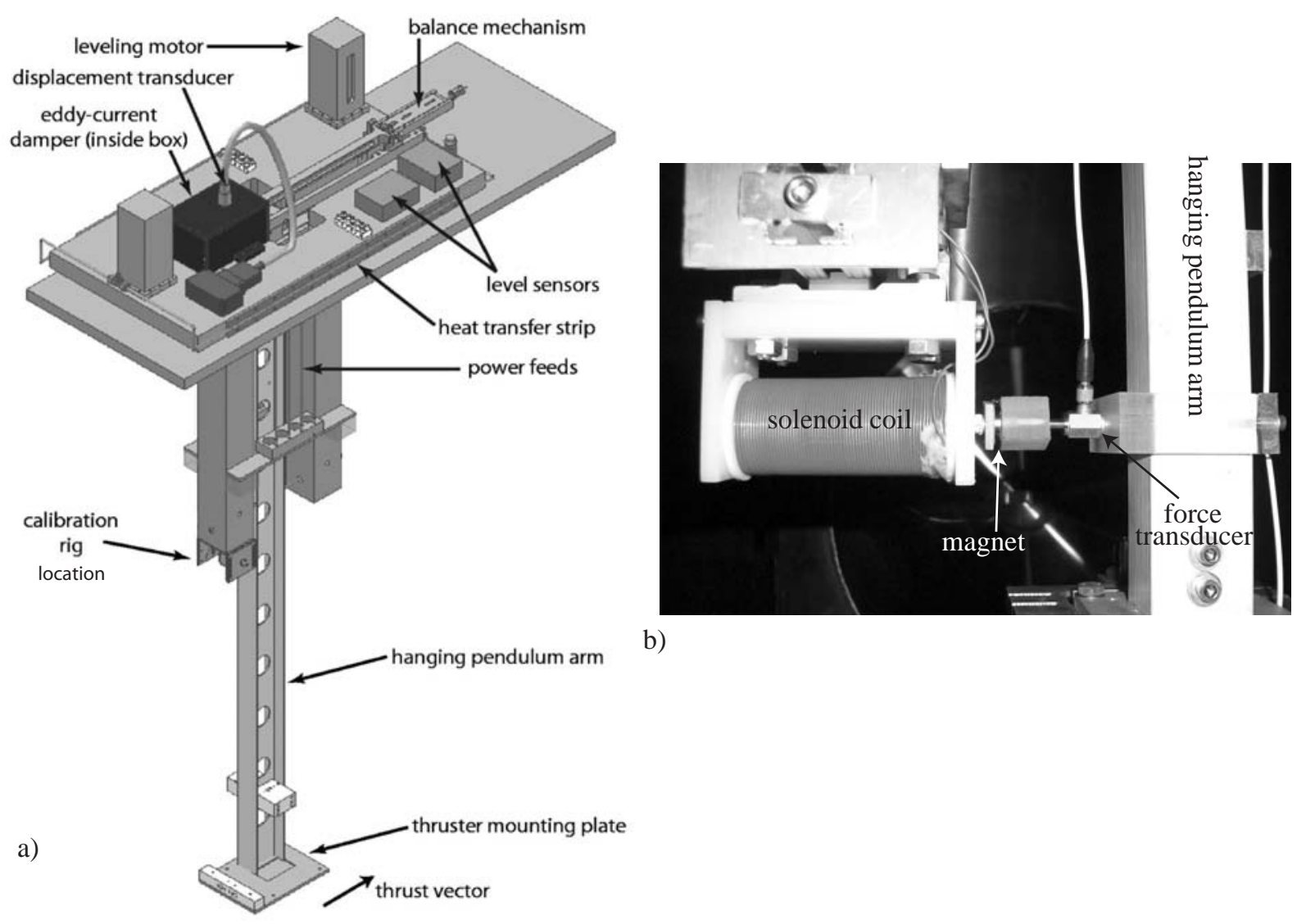

Figure 3. a) Schematic of the VAHPER thrust stand. b) Photograph of the pulsed calibration apparatus mounted on the thrust stand.

trial are presented in Fig. 4. These data were acquired for a repetition rate of $10 \mathrm{~Hz}$ with force applied for $50 \mathrm{ms,} \mathrm{with}$ active pulsing over $30 \mathrm{~s}$ to reach a steady-state value. We observe that that when pulsing is initiated at $t=0$ the thrust stand deflects and oscillates as expected for a spring-mass-damper system until reaching a near-steady value beyond roughly $10 \mathrm{~s}$. Once the forcing is discontinued, the same damped sinusoidal trend is observed about the zero deflection point. The difference between the zero position and the quasi-steady long term deflection during pulsing is taken as the average deflection for a measurement.

Corresponding to the low-speed data in Fig. 4 are high-speed measurements in Fig. 5a of a single pulsed force applied to deflect the thrust stand arm. Temporally, this pulse occurs near the end of the pulsing time (near $t=30 \mathrm{~s}$ in Fig. 4). This force is integrated with respect to time to yield the impulse applied during the pulse as presented in Fig. $5 \mathrm{~b}$, with the maximum value at approximately $50 \mathrm{~ms}$ taken as the impulse bit $I_{\text {bit }}$. We observe a $333 \mathrm{~Hz}$ oscillation in the force measurement, which are real oscillations in the applied force caused by current oscillations in the inductive solenoid coil owing to impedance mismatches in the electrical system. After integration the effect of this oscillation on the impulse is approximately zero. The average force applied to the thrust stand during a pulse train is simply the product of $I_{\mathrm{bit}}$ and the pulse frequency.

Calibration data are presented in Fig. 6a for a pulse frequency held constant at $10 \mathrm{~Hz}$ while the current through the solenoid coil, and consequently the applied force per pulse, is varied from trial to trial. In Fig. $6 \mathrm{~b}$ the pulse frequency is varied in each trial from 1 to $10 \mathrm{~Hz}$. The pulse width is $50 \mathrm{~ms}$ for all the data presented in Fig. 6 . We observe that the average thrust as a function of average deflection is quite linear for both variable applied force and variable pulse frequency. More importantly, the linear calibration constant $k_{\text {eff }}$ in both cases is the same, within the calculated error on the regression fit, demonstrating the calibration technique described in Sect. II and showing the ability of the test apparatus to apply a wide range range of average forces to the thrust stand. The calibration constant errors for these data are roughly $1 \%$ of the total value of $k_{\text {eff. }}$. Data was acquired in Fig. $6 \mathrm{~b}$ only down to $1 \mathrm{~Hz}$ since below this level the stand never reached a steady-state deflection following the linear trend but instead exhibited chaotic behavior, as was illustrated by the low-frequency modeling results in Fig. $2 b$. 


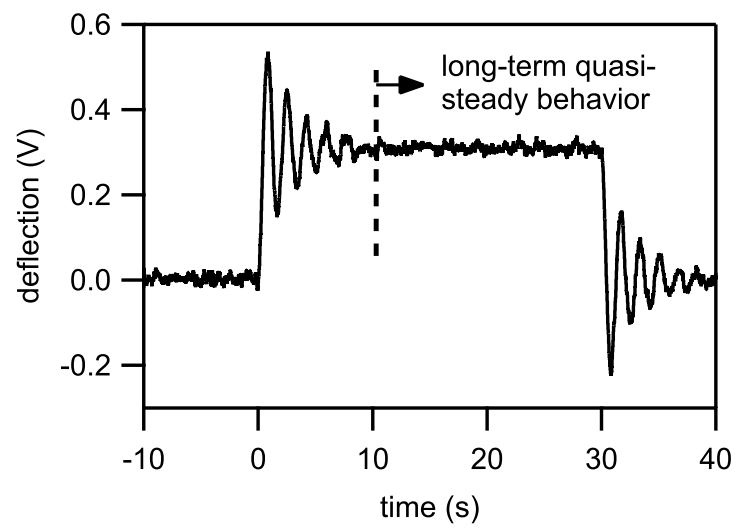

Figure 4. Low-speed data showing the time-history of thrust stand deflection (given in $\mathrm{V}$ ) during repetition-rate pulsing at a rate of $10 \mathrm{~Hz}$ over a 30 s timespan.
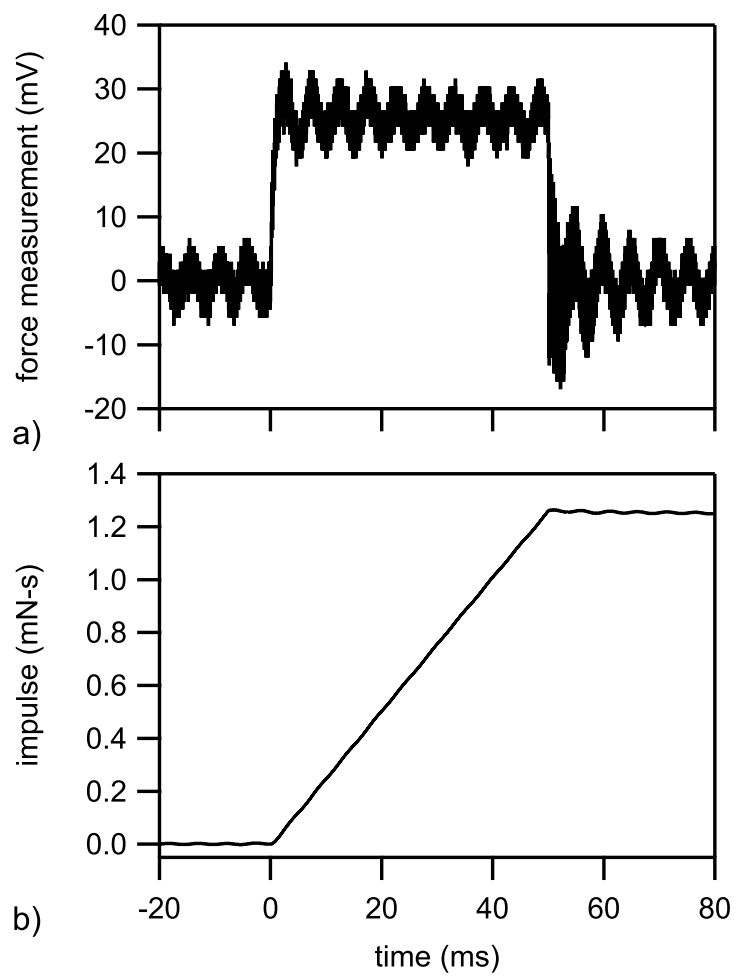

Figure 5. High-speed data showing a) the measured force and b) the impulse bit for a single $\mathbf{5 0}$ ms pulse driving the response of the thrust stand as shown in Fig. 4.

\section{Conclusions}

We have developed a non-contact thrust stand calibration technique for use in testing pulsed electric thrusters for in-space propulsion. The force is applied in a non-contact manner to a pendulum-based thrust stand by pulsing current through a solenoid coil that produces a pulsed magnetic field pushing against a permanent magnet mounted to the thrust stand pendulum arm. The force on the magnet is transferred to the pendulum arm through a piezoelectric force transducer, providing a real-time measurement of the force applied in a pulse. Unlike previous pulsed electric thruster 

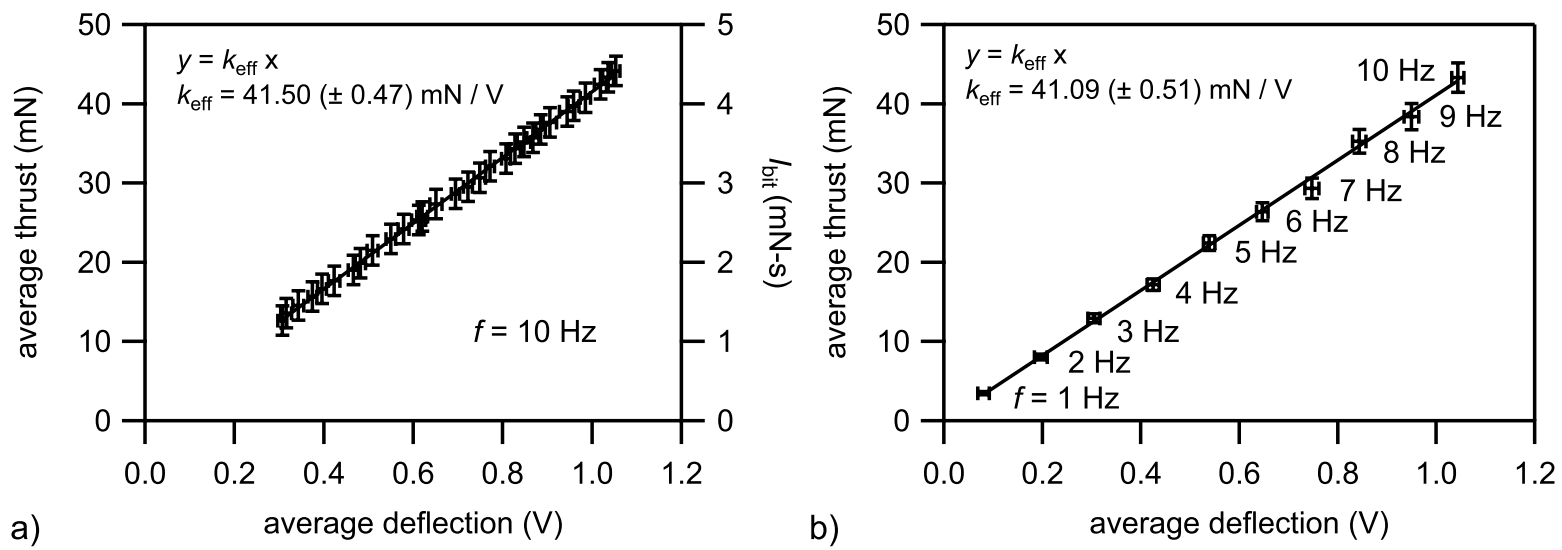

Figure 6. Measured average force applied to the system as a function of the measured average long-term deflection for a) varying impulse bit at a pulse frequency of $10 \mathrm{~Hz}$ and b) varying pulse frequency.

work that focused primarily on single-shot pulsed measurements, this technique described in this paper has been developed for use on pulsed thrusters operating in a repetition-rate mode. Dynamic thrust stand modeling shows that after an initial transient in thrust balance deflection the average deflection away from the unforced or 'zero' position can be linearly related to the average pulsed force. This yields a simple linear Hooke's law relationship between average displacement and the thrust where the spring constant $k_{\text {eff }}$ is the same as the one governing the dynamic motion of the thrust stand. The success of this method relieves the requirement to perform a more highly resolved temporal deflection measurement during repetition-rate pulsed thrust measurements in cases where the deflection during pulsing reaches a steady-state value. The method is not valid for pulse rates below a given frequency as the deflection never reaches a steady-state value.

The technique was tested using a modified hanging pendulum thrust stand that had previously been used to obtain high-resolution steady-state thrust measurements on low-power Hall thrusters. Calibration data were acquired first by varying the impulse bit at constant frequency by varying the solenoid current, and consequently the magnetic field repelling the permanent. A second set of calibration data were acquired by varying the average thrust by changing pulse frequency at a relatively constant solenoid current. The linear calibration constant $k$ eff obtained from both methods matched within the calculated error on the linear regression fit, and the overall error on the constant was roughly $1 \%$ of the coefficient value.

\section{Acknowledgments}

We appreciate the continued management support of Mr. Jim Martin and Ms. Mary Beth Koelbl and the continued academic affairs support of Ms. Mona Miller and Ms. Tina Haymaker. We gratefully acknowledge the contributions and support during the course of this effort by Tommy Reid, Doug Galloway, Ashley Hallock, Derek Mayer, Adam Kimberlin, and Kevin Bonds. This program was supported under NASA's Advanced In-Space Propulsion program managed by Dr. Michael LaPointe.

\section{Appendix: Solution to the thrust stand deflection initial value problem}

The following is a derivation of the solution to the initial value problem of Eq. (1) subject to the initial conditions $\dot{x}(t)=x(t)=0$. The Laplace transform of the governing differential equation:

$$
\mathcal{L}\left[\ddot{x}(t)+2 \omega_{n} \zeta \dot{x}(t)+\omega_{n}^{2} x(t)=\sum_{i=0}^{k} \frac{I_{\mathrm{bit}}}{m_{\mathrm{eff}}} \delta\left(t-\frac{i}{f}\right)\right]
$$


results in

$$
\left[s^{2} X(s)-x(0) s-\dot{x}(0)\right]+2 \omega_{n} \zeta[s X(s)-x(0)]+\omega_{n}^{2} X(s)=\sum_{i=0}^{k} \frac{I_{\mathrm{bit}}}{m_{\mathrm{eff}}} \exp (-s(i / f))
$$

where $s$ is the transform variable. Under the initial conditions, the equation can be rearranged as

$$
X(s)=\sum_{i=0}^{k} \frac{I_{\mathrm{bit}}}{m_{\mathrm{eff}}} \frac{\exp (-s(i / f))}{s^{2}+2 \omega_{n} \zeta s+\omega_{n}^{2}}
$$

The inverse Laplace transform of this equation yields the solution, which is given as

$$
\begin{array}{cc}
x(t)=\sum_{i=0}^{k}\left[\frac { I _ { \mathrm { bit } } } { 2 m _ { \mathrm { eff } } \omega _ { n } \sqrt { \zeta ^ { 2 } - 1 } } \operatorname { e x p } \{ - \omega _ { n } \zeta ( t - \frac { i } { f } ) \} \left(\exp \left\{\omega_{n} \sqrt{\zeta^{2}-1}\left(t-\frac{i}{f}\right)\right\}\right.\right. & \text { for } \zeta>1 \\
\left.\left.-\exp \left\{-\omega_{n} \sqrt{\zeta^{2}-1}\left(t-\frac{i}{f}\right)\right\}\right)\right] H\left(t-\frac{i}{f}\right) & \text { for } \zeta=1 \\
x(t)=\sum_{i=0}^{k}\left[\frac{I_{\mathrm{bit}}}{m_{\mathrm{eff}}}\left(t-\frac{i}{f}\right) \exp \left\{-\omega_{n}\left(t-\frac{i}{f}\right)\right\}\right] H\left(t-\frac{i}{f}\right) & i \\
x(t)=\sum_{i=0}^{k}\left[\frac{I_{\mathrm{bit}}}{m_{\mathrm{eff}} \omega_{n} \sqrt{1-\zeta^{2}}} \exp \left\{-\omega_{n} \zeta\left(t-\frac{i}{f}\right)\right\} \sin \left\{\omega_{n} \sqrt{1-\zeta^{2}}\left(t-\frac{i}{f}\right)\right\}\right] H\left(t-\frac{i}{f}\right)
\end{array}
$$

where $H$ is the Heaviside function. Note that these equations match those given in Eqs. (2)-(4) for $\dot{x}(t)=x(t)=0$. The general solution given in those equations can be obtained by following the same steps while keeping the general initial position and velocity terms in Eq. (A2).

\section{References}

\footnotetext{
${ }^{1}$ Y. Lee, G.N. Kudva, and T.A. Litzinger, "A facility for solid-propellant response measurements under pressure-driven conditions," Meas. Sci. Technol., 11, 51 (2000).

${ }^{2}$ E.A. Cubbin, J.K. Ziemer, E.Y. Choueiri, and R.G. Jahn, "Pulsed thrust measurements using laser interferometry," Rev. Sci. Instrum. 68, 2339 (1997).

${ }^{3}$ T.W. Haag, "Thrust stand for pulsed plasma thrusters," Rev. Sci. Instrum., 68, 2060 (1997).

${ }^{4}$ T.E. Markusic, K.A. Polzin, E.Y. Choueiri, M. Keidar, I.D. Boyd, and N. Lepsetz, “Ablative Z-pinch pulsed plasma thruster,” J. Propuls. Power 21, 392 (2005).

${ }^{5}$ K.A. Polzin, T.E. Markusic, B.J. Stanojev, A. Dehoyos, and B. Spaun, "Thrust stand for electric propulsion performance evaulation," Rev. Sci. Instrum., 77, 105108 (2006).

${ }^{6}$ K.A. Polzin, T.E. Markusic, B.J. Stanojev, A. DeHoyos, Y. Raitses, A. Smirnov, and N.J. Fisch, "Performance of a low-power cylindrical Hall thruster," J. Propuls. Power 23, 886 (2007).

${ }^{7}$ K.A. Polzin, Y. Raitses, J.C. Gayoso, and N.J. Fisch, "Comparisons in performance of electromagnet and permanent-magnet cylindrical Hall-effect thrusters," 46th AIAA/ASME/SAE/ASEE Joint Propulsion Conference, Nashville, TN, July 2010. AIAA Paper 2001-6695.
} 